\title{
Effect of nanomaterials on the absorbed dose during an X-ray exposure
}

\author{
F. Benlakhdar, A.S.A. Dib ${ }^{\star}$ and A.H. Belbachir \\ Université des Sciences et de la Technologie d'Oran Mohamed Boudiaf, USTO-MB, BP 1505 EL M'naouer, 31000 Oran, Algeria.
}

Received 6 April 2016 - Accepted 20 September 2016

\begin{abstract}
This work is based on a numerical study using the Monte Carlo code Geant 4 of an X-ray exposure of a human head containing a tumor at the center. Our principal goal was to investigate the effect of inserting nanomaterials into the tumor on the absorbed dose. Gold is the most popular nanomaterial used in nanomedicine. In our simulation, we also focused on the other nanomaterials platinum, gadolinium and silver. Our results show that gold and platinum increase the absorbed dose in the tumor by up to 55\%; the most suitable X-ray energy is between $20 \mathrm{keV}$ and $140 \mathrm{keV}$. Both of these nanomaterials present the same advantages in X-ray therapy. Moreover, gadolinium increases the absorbed dose by up to $50 \%$, while silver increases the absorbed dose by up to $20 \%$. However, for both of these nanomaterials, the best X-ray energy to use is between $20 \mathrm{keV}$ and $80 \mathrm{keV}$.
\end{abstract}

Keywords: Radiation dose / human organ / tumors / X-ray

\section{Introduction}

Nowadays, nanotechnology in cancer treatments has become a reality, providing new tools, from earlier diagnostics and improved imaging to better, more efficient, and more targeted therapies. One of the most promising ways to achieve this goal is the use of bionanomaterials. In recent years, the in vitro and in vivo biocompatibility of gold nanomaterials has been examined in depth (Connor et al., 2005; Brun et al., 2008; Boisselier and Astruc, 2009); because of its high atomic number $(\mathrm{Z})$ and strong photoelectric coefficient, gold has been investigated as a radiosensitizing nanomaterial inside cancer cells to improve the dose in radiotherapy (Herold et al., 2000; Cho, 2005). An ideal radiation therapy treatment delivers a sufficiently high dose to cancerous cells without adverse effects to normal tissues. In this field, many studies have suggested using Monte Carlo simulation (Casta et al., 2014; Berrezoug et al., 2015). These studies explain the increase in destruction of cancerous cells by the electrons emitted from nanoparticles (Butterworth et al., 2008; Brun et al., 2009). Theoretical studies were characterized to evaluate numerous aspects of the application of gold nanoparticles for external beam radiotherapy or brachytherapy (Bahreyni Toossi et al., 2012; Delaram et al., 2013). Their capacity to absorb high quantities of X-ray radiation can be used to enhance cancer radiation therapy or increase imaging contrast in diagnostic computed tomography (Sanche, 2009). The application of Monte Carlo code in radiotherapy has been validated by several researchers. Calculation of the depth dose was evaluated by Noblet et al. (2016), who

\footnotetext{
^ dibanis70@gmail.com
}

confirmed good agreement between simulations and measurements, with uncertainties estimated at $1 \%$.

In a MC study, the interaction of X-rays with single gold nanoparticles (GNPs) with different diameters was considered. It was shown that larger sizes of nanoparticle offer a higher dose (Leung et al., 2011). In other research, the energy deposition due to secondary electrons produced from GNPs and the microscopic dose enhancement around GNPs were quantified (Jones et al., 2010). The MC results obtained by McMahon (McMahon et al., 2011; Lechtman et al., 2011, 2013; GarnicaGarza et al., 2013) are in good agreement with experimentally observed cell killing by the combination of X-rays and GNPs. In an experimental study (Rahman et al., 2009) where kilovolt X-ray radiation and megavolt electron radiation were used in the presence of GNPs in bovine aortic endothelial cells, it was found that the cell damage increases with the increase in the GNP concentration. Nanoparticles (NPs) can be delivered orally or intravenously. Because tumor cells are bigger and more numerous than normal cells, they have the capacity to consume more substances. Therefore, each tumor cell has the ability to contain more than one NP. Several studies have investigated the effects of inserting GNPs into tumor cells (Chow et al., 2012; Ricketts et al., 2012).

In practice, X-rays are generally used as a radiosensitizer with the use of the biomarker agent for diagnosis of tumors. In this paper, we are interested in the effect of some nanomaterials on the absorbed dose. Therefore, we focused our simulation on platinum, gadolinium, silver and gold, the most popular nanomaterials in nanomedicine research. Consequently, our study is composed of two parts; the first part is devoted to a simulation of a human head exposed to X-rays. The principal goal 


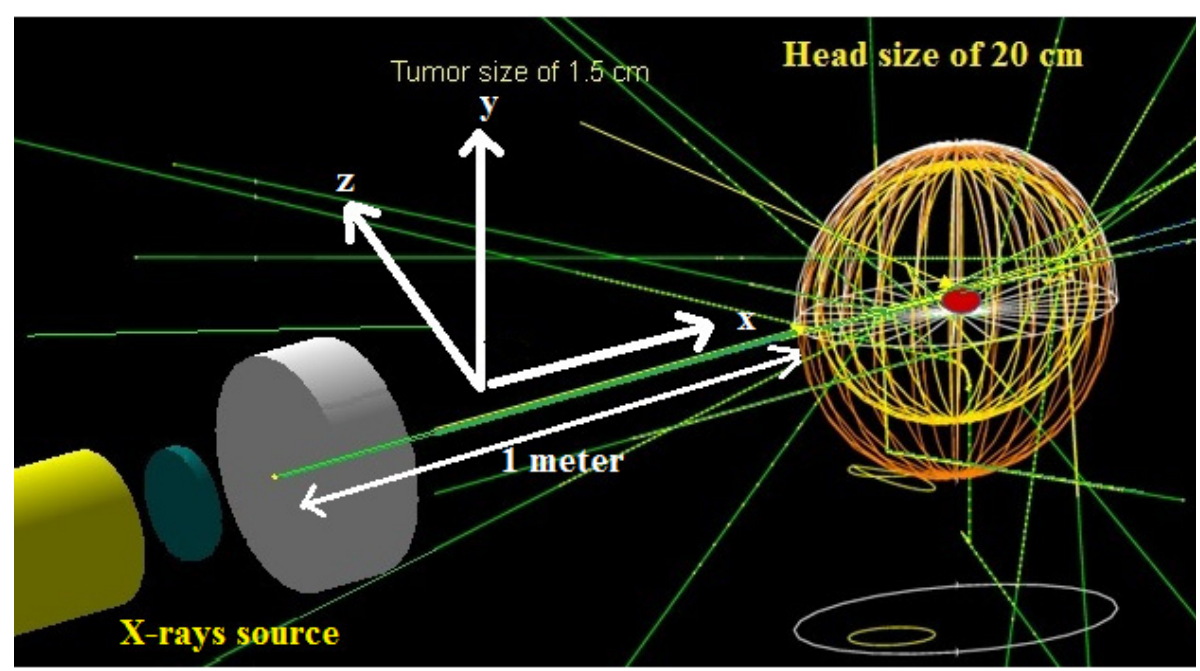

Figure 1. Monte Carlo simulation of X-ray radiation beam interaction with a human head. The radiation source is placed 1 meter from the head. The energy of the radiation beam ranged between $20 \mathrm{keV}$ and $200 \mathrm{keV}$.

of this simulation is to investigate the effect of inserting nanomaterials on the absorbed dose in a tumor inside a head. The second part of our study investigates in depth the X-ray beam interaction with a nanoparticle inside a water box.

\section{Methods and geometry}

Our main study was to investigate the effect of bionanomaterials (bioNMs) injected into a tumor during X-ray radiation. We simulated a spherical tumor localized in the center of a human head. Then, the human head was exposed to isotropic $\mathrm{X}$-ray energy ranging between $20 \mathrm{keV}$ and $200 \mathrm{keV}$. Like most medical X-ray scanners used in radiation therapy, the radiation source is placed 1 meter from the patient (see Figure 1).

\subsection{Monte Carlo simulations}

Geant4 (Agostinelli et al., 2003; Allison et al., 2006) is a platform for the simulation of the passage of particles through matter using Monte Carlo methods. It is used in various application domains, including high energy physics, astrophysics and space science, and medical physics. In this work, the low electromagnetic energy package was used together with the Geant4-DNA extensions (Chauvie et al., 2007; Incerti et al., 2016).

\subsection{Materials and geometry}

\subsubsection{Simulation of a tumor inside a human head}

In this part of our paper, we carried out a Monte Carlo simulation of an X-ray exposure of a tumor inside a human head; we were interested in calculating the absorbed dose in the tumor with and without nanoparticles. The tumor was assumed to have a spherical shape with a diameter of $1.5 \mathrm{~cm}$, and to be localized at the center of the head (see Figure 1). $1.5 \mathrm{~cm}$ for the diameter of the tumor was chosen so as to see the effect of inserting nanomaterials into a deep tumor inside a human head. The best way to inject NMs into a tumor localized in a sensitive area such as the human head is by the bloodstream. Several teams (Carmeliet and Jain, 2000; Avnesh et al., 2013) studied the development of blood vessels in tumor cells; they noted that the blood vessels are more concentrated in the center of a tumor. Consequently, nanomaterials were distributed inside the tumor in a non-homogeneous way and concentrated more toward the center of the tumor. In this simulation, the concentration of nanomaterials in the tumor was assumed to range between 0 and $4 \%$, and they are not present outside the tumor.

The geometry of the human head is composed essentially of a skeleton of $0.8 \mathrm{~cm}$ thickness and brain matter. Then, a soft $0.2-\mathrm{cm}$-thick tissue covers the skeleton. The tumor is placed in the center of the head. The chemical compositions and densities of the skeleton, brain, soft tissue and tumor are taken from the Geant4 database (see Table 1).

Figure 2 shows the plot of the absorbed dose in the head when exposed to X-rays of $80 \mathrm{keV}$. In this plot, the human head is exposed to an X-ray source placed $1 \mathrm{~m}$ from the patient. In this part of our simulation, we used the low electromagnetic energy package with cut-off energy of $250 \mathrm{eV}$ and the step range of $0.01 \mathrm{~mm}$. To obtain results with good accuracy, $10^{8}$ gamma rays were emitted from the radiation source; the calculation time took more than three days on a HP Z800 Workstation. This figure shows that the absorbed dose increases considerably with the insertion of GNPs into the tumor. For more details, Figure 3 presents the calculated absorbed dose in the tumor resulting from the addition of different NMs at the same concentration and during an X-ray exposure with the energy ranging between $20 \mathrm{keV}$ and $200 \mathrm{keV}$. As can be seen, in this $\mathrm{X}$-ray energy range the plots of the absorbed dose in the tumor when adding gold or platinum NPs are similar and present two peaks. The first peak occurs for X-ray energy around $50 \mathrm{keV}$. At this energy value, the absorbed dose is slightly higher when adding gold NPs rather than platinum NPs. The second peak 
Table 1. Chemical compositions and densities of the materials used in our geometry.

\begin{tabular}{llll}
\hline \multicolumn{1}{c}{ Materials and densities } & \multicolumn{3}{c}{ Chemical compositions } \\
\hline Soft tissue $\left(0.9869 \mathrm{~g} \mathrm{~cm}^{-3}\right)$ & $\mathrm{H}(10.47 \%) ; \mathrm{C}(23.02 \%) ; \mathrm{N}(2.34 \%) ; \mathrm{O}(63.21 \%) ; \mathrm{Na}(0.1 \%) ; \mathrm{Mg}(0.015 \%) ;$ \\
& $\mathrm{P}(0.24 \%) ; \mathrm{S}(0.22 \%) ; \mathrm{Cl}(0.14 \%) ; \mathrm{K}(0.2 \%) ; \mathrm{Ca}(9.91 \%) ; \mathrm{Fe}(0.0063 \%) ;$ \\
& $\mathrm{Zn}(0.0032 \%) ; \mathrm{Rb}(0.00057 \%) ; \mathrm{Sr}(0.000034) ; \mathrm{Pb}(0.000016 \%) ; \mathrm{Zr}(0.00008 \%)$ \\
Skeleton $\left(1.4862 \mathrm{~g} \mathrm{~cm}^{-3}\right)$ & $\mathrm{H}(7.04 \%) ; \mathrm{C}(22.79 \%) ; \mathrm{N}(3.87 \%) ; \mathrm{O}(48.56 \%) ; \mathrm{Na}(0.32 \%) ; \mathrm{Mg}(0.11 \%) ;$ \\
& $\mathrm{P}(6.94 \%) ; \mathrm{S}(0.17 \%) ; \mathrm{Cl}(0.14 \%) ; \mathrm{K}(0.15 \%) ; \mathrm{Ca}(9.91 \%) ; \mathrm{Fe}(0.008 \%) ;$ \\
& $\mathrm{Zn}(0.0048 \%) ; \mathrm{Sr}(0.0032 \%) ; \mathrm{Pb}(0.0011 \%)$ \\
Brain $\left(1.040 \mathrm{~g} \mathrm{~cm}^{-3}\right)$ & $\mathrm{H}(10.70 \%) ; \mathrm{C}(14.50 \%) ; \mathrm{N}(2.20 \%) ; \mathrm{O}(71.20 \%) ; \mathrm{Na}(0.20 \%) ; \mathrm{P}(0.40 \%) ;$ \\
& $\mathrm{S}(0.20 \%) ; \mathrm{Cl}(0.30 \%) ; \mathrm{K}(0.30 \%)$ \\
Tumor $\left(1.256 \mathrm{~g} \mathrm{~cm}^{-3}\right)$ & $\mathrm{H}(10.70 \%) ; \mathrm{C}(14.50 \%) ; \mathrm{N}(2.20 \%) ; \mathrm{O}(71.20 \%) ; \mathrm{Na}(0.20 \%) ; \mathrm{P}(0.40 \%) ;$ \\
& $\mathrm{S}(0.20 \%) ; \mathrm{Cl}(0.30 \%) ; \mathrm{K}(0.30 \%)$ \\
\hline
\end{tabular}

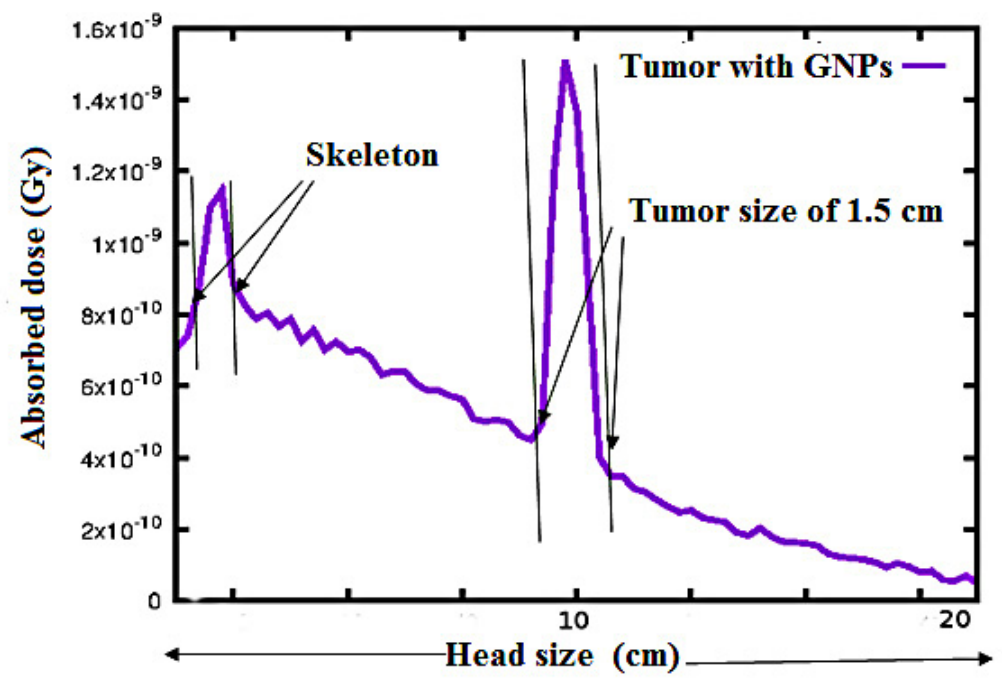

Figure 2. The absorbed dose in the head during head irradiation using an $80-\mathrm{keV} \mathrm{X}$-ray beam.

occurs for X-ray energy of about $80 \mathrm{keV}$ in the case of platinum NPs and about $85 \mathrm{keV}$ in the case of gold NPs. The plots of the absorbed dose in the tumor when adding silver or gadolinium NPs present a maximum for X-ray energy around $50 \mathrm{keV}$. In Figure 3, it can be seen that both gold and platinum nanomaterials present the same advantages. Their insertion into the tumor results in an increase in the absorbed dose in the tumor of more than $55 \%$. On the other hand, gadolinium enhances the absorbed dose in the tumor by up to $50 \%, 2.5$ times more than silver. Comparing the effect of these nanomaterials on the absorbed dose in the tumor, gold and platinum nanomaterials increase the absorbed dose significantly, especially in the X-ray energy ranging from about $40 \mathrm{keV}$ to $150 \mathrm{keV}$. However, gadolinium, gold and platinum nanomaterials are more efficient than silver in the X-ray energy ranging between $40 \mathrm{keV}$ and $80 \mathrm{keV}$. To explain the results found in Figure 3, we suggest investigating the effect of nanomaterials used previously with an isotropic source X-ray on a microscopic scale.

\subsubsection{Simulation of one type of nanomaterial inside a water box}

In this part of our work, we studied the interaction of an $\mathrm{X}$-ray beam with a water box containing one type of nanopar- ticle. The water box size was $1 \mathrm{~mm}^{3}$ and the nanomaterial particle diameter was taken to be $10 \mathrm{~nm}$ (see Figure 4). The simulation was based on the Geant4_DNA package (Chauvie et al., 2007; Incerti et al., 2016). The step range was taken to be 1 nanometer, and the radiation beam $10^{8}$ gamma. In this case, the calculation time took $24 \mathrm{~h}$ on a HP Z800 Workstation. The interaction of X-rays with metallic nanoparticles generates several physical processes; some of these processes are photoelectric effects, ionization and electron multiscattering based on Goudsmit-Saunderson angular distribution and the Lewis spatial displacement theories (Kadri et al., 2009).

The radiation beam is emitted in the $\mathrm{x}$-axis, and the presence of one type of nanomaterial inside the water box generates secondary electrons and photons. As Figure 4 shows, the green lines define secondary X-rays, and the red lines define secondary electrons. The yellow dots represent the step range of secondary electrons and photons. For an external Xray energy of $100 \mathrm{keV}$, the step range of secondary electrons is around a few micrometers outside the GNP.

In particular, for heavy atoms, the Auger effect is the most interesting physical process in this interaction. An Auger electron is ejected from the inner electron shell of the metal, which creates a vacancy. When an upper electron fills the vacancy, this gives rise to the emission of a photon, called X-ray fluorescence. The Auger electron spectrum is represented in 


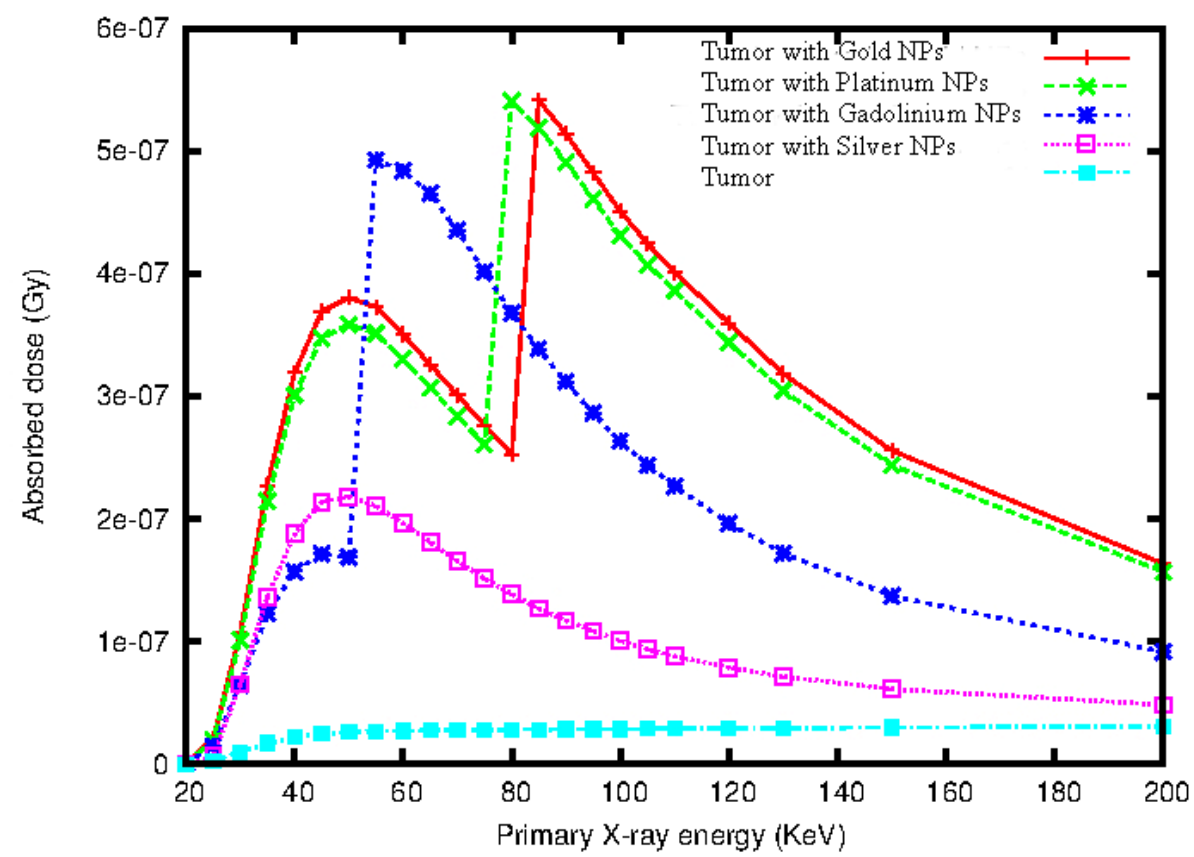

Figure 3. Plots of the absorbed dose within the tumor versus the X-ray energy (ranging from $20 \mathrm{keV}$ to $200 \mathrm{keV}$ ) for different types of NPs with a non-homogeneous concentration ranging between 0 and $4 \%$. The tumor is localized within the brain.

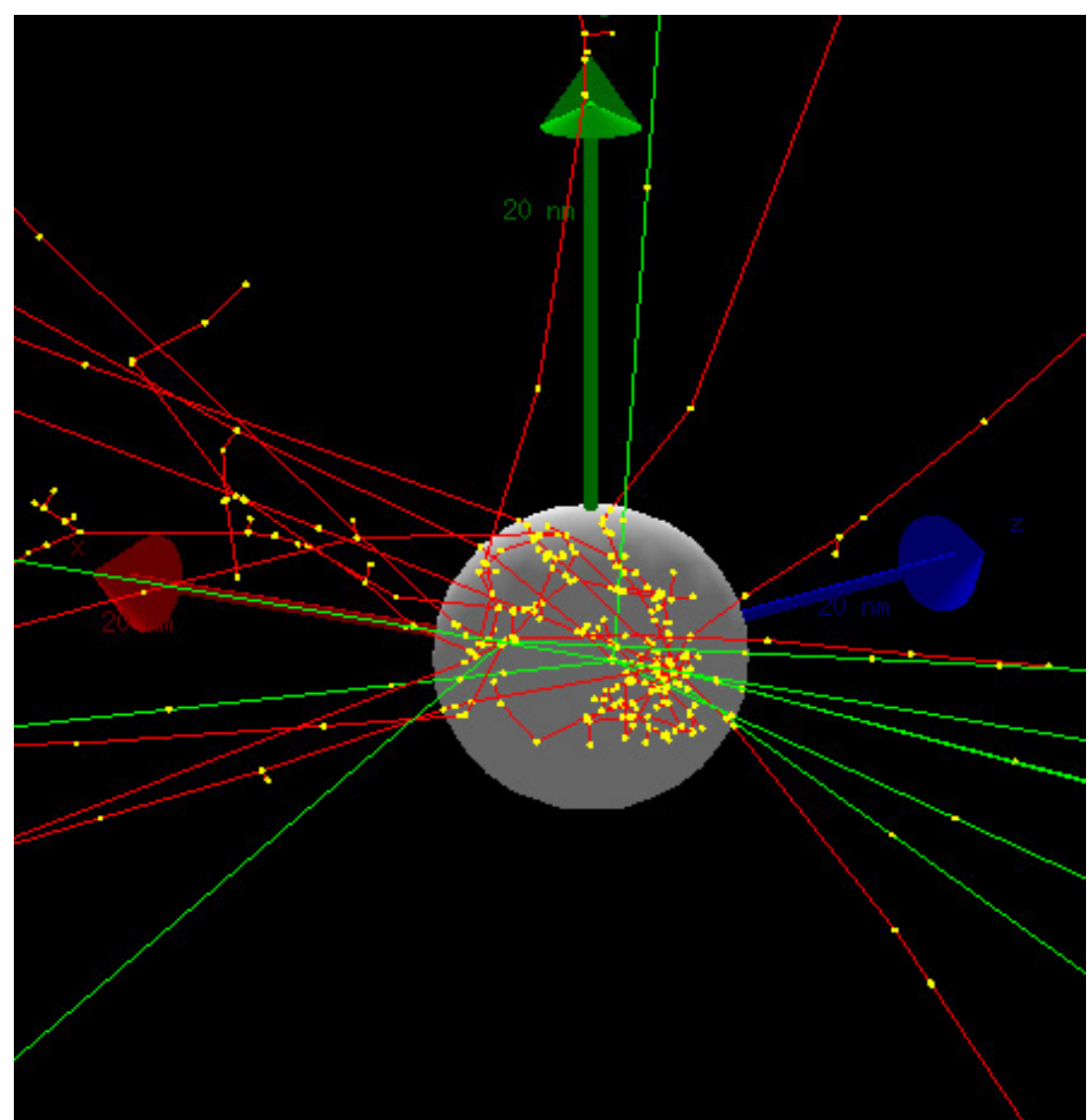

Figure 4. Simulation of X-ray beam interaction with a spherical GNP of a diameter of 10 nanometers in a water box with sides of $1 \mathrm{~mm}^{3}$. The green lines define secondary X-rays, and the red lines define secondary electrons. The yellow dots represent the step range of secondary electrons. In this figure the X-ray energy is $100 \mathrm{keV}$. 


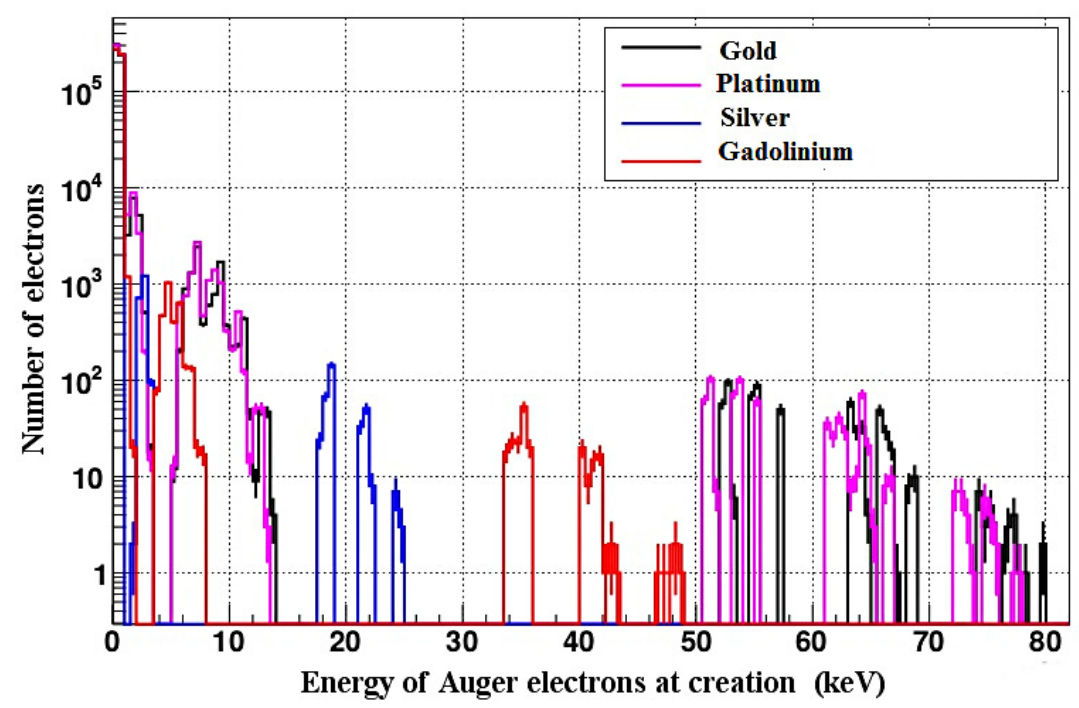

Figure 5. Spectrum of Auger electrons at an X-ray energy of $100 \mathrm{keV}$.

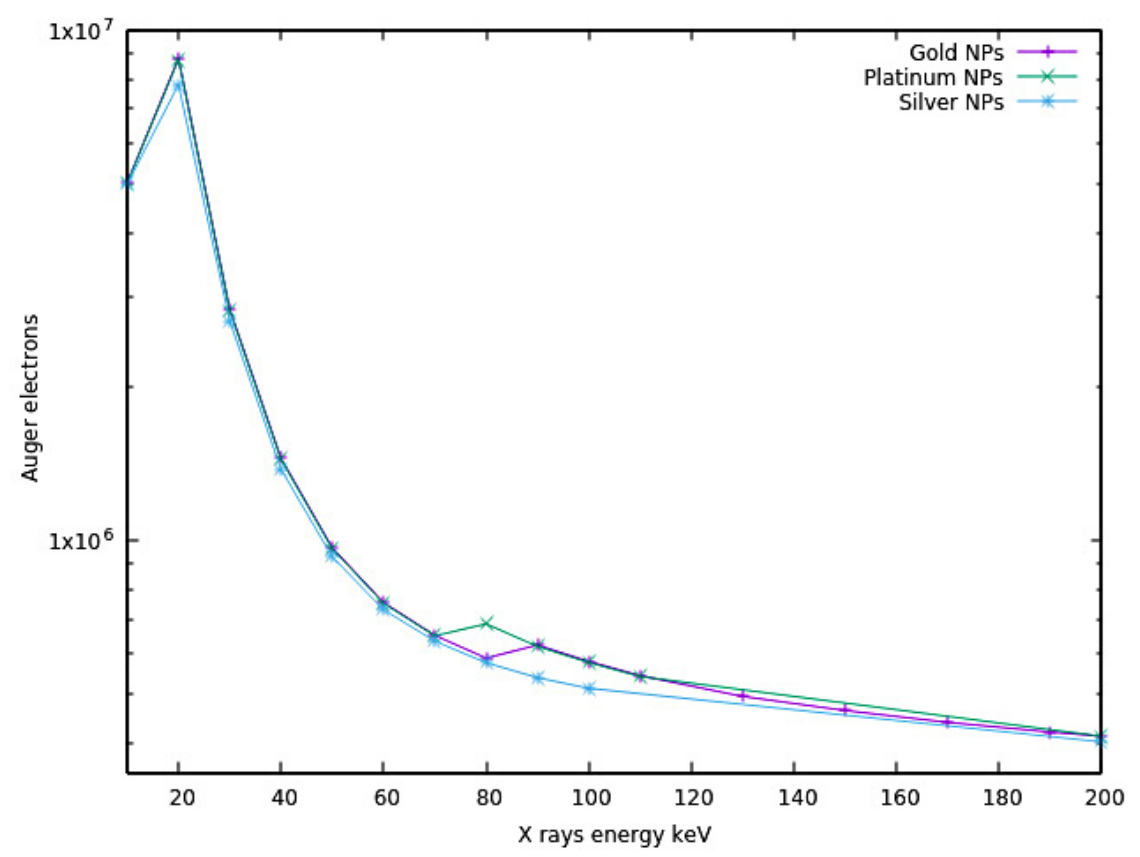

Figure 6. Number of Auger electrons produced by X-ray energy ranging from $10 \mathrm{keV}$ to $200 \mathrm{keV}$.

Figure 5 in the case of different metallic nanoparticle types. In this figure, it can be seen that platinum and gold nanomaterials present the same Auger electron spectrum below $20 \mathrm{keV}$, and slightly shifted in the energy range between $50 \mathrm{keV}$ and $80 \mathrm{keV}$. These spectra clearly show the existence of two energy peaks in Figure 3 in the case of gold and platinum nanomaterials. Furthermore, gadolinium presents two regions of discrete energy; the first is below $9 \mathrm{keV}$ and the second is between $35 \mathrm{keV}$ and $50 \mathrm{keV}$. Silver presents an energy spectrum of the electrons emitted ranging between $15 \mathrm{keV}$ and $25 \mathrm{keV}$. Moreover, we plotted the number of emitted Auger electrons as a function of the X-ray energy (see Figure 6). As can be seen, this plot presents a maximum number of emitted Auger electrons at $20 \mathrm{keV}$. This result is not in contradiction with the previous results in Figure 3; with the presence of nanomaterials, the absorbed dose increases in function of the energy of secondary electrons and photons. As Figure 5 shows, gold and platinum nanomaterials are the most favorable to enhance the dose absorption inside a tissue, with a slight difference between them.

\section{Results and discussion}

Over the last few decades, a large number of nanomaterial delivery systems have been developed for radiation therapy, including organic and inorganic materials. Many nanoparticles are currently in the preclinical stages of development. The use 
of gold nanoparticles in radiation therapy is increasing exponentially, especially in imaging and tumor diagnosis applications, because of their biocompatibility and ability to convert energy radiation into heat (Abadeer et al., 2016). In this paper, we carried out a Monte Carlo simulation based on the Geant4 code of an external X-ray beam irradiating a human head tumor, and we investigated the effect of inserting metal nanoparticles into this tumor in order to enhance this therapy.

Our results show that the presence of gold or platinum nanomaterials in the tumor considerably increases the absorbed dose in the tumor (Figures 2 and 3). Indeed, a density of $1 \%$ of these nanomaterials in the tumor is enough to enhance the absorbed dose by up to $55 \%$. In order to increase the absorbed dose in the case of a deep tumor, as considered in our simulation, with the presence of nanoparticles in the tumor, the energy of the X-ray should be between $40 \mathrm{keV}$ and $120 \mathrm{keV}$. The Auger electron spectrum (Figure 5) clearly shows that both gold and platinum nanomaterials interact in the same way during an X-ray exposure. Gadolinium and silver nanomaterials were also considered in this study. Gadolinium enhances the absorbed dose by up to $50 \%$, while silver enhances the absorbed dose by up to $20 \%$. As Figure 3 shows, with the use of these two nanomaterials, the absorbed dose is more enhanced in the X-ray energy range between $40 \mathrm{keV}$ and $80 \mathrm{keV}$.

Our simulation results show that to increase the dose absorption in a deep tumor inside a sensitive area such as the human head, gold and platinum are the most appropriate for use during X-ray therapy. Furthermore, inserting a density of $1 \%$ of these nanomaterials inside a tumor with a size of $1.5 \mathrm{~cm}$ is sufficient to increase the absorbed dose by more than $55 \%$. However, to avoid toxicity and adverse effects when using these nanometals, we propose to study in a future work the relationship between the tumor size and the percentage of nanomaterials to insert into the tumor.

\section{References}

Abadeer N.S., Murphy C.J. (2016) Recent Progress in Cancer Thermal Therapy Using Gold Nanoparticles, J. Phys. Chem. C 120 (9), 4691-4716.

Agostinelli S. et al. (2003) Geant4- a simulation toolkit, Nucl. Instrum. Methods Phys. Res. A 506, 250-303.

Allison J. et al. (2006) Geant4 developments and applications, IEEE Transactions on Nuclear Science 53, 270-278.

Avnesh S., Thakor M.D., Sanjiv S., Gambhir, M.D. (2013) Nano oncology: The future of cancer diagnosis and therapy, CA: A Cancer Journal for Clinicians, DOI:10.3322/caac.21199.

Bahreyni Toossi M.T. et al. (2012) A Monte Carlo study on tissue dose enhancement in brachytherapy: a comparison between gadolinium and gold nanoparticles, Australas. Phys. Eng. Sci. Med. 35, 177-185.

Berrezoug A., Dib A.S.A., Belbachir A.H. (2015) Enhanced X-ray absorption by using gold nanoparticles in a biological tissue, Radioprotection 50 (4), 281-285.

Boisselier E., Astruc D. (2009) Gold nanoparticles in nanomedicine: preparations, imaging, diagnostics, therapies and toxicity, Chem. Soc. Rev. 38, 1759-1782.

Brun E., Simon-Deckers A., Carriere M., Sanche L., Sicard-Roselli C. (2008) Experimental Evidence of Gold Nanoparticle Radiosensitization in vitro, Radioprotection 43 (5), 185.
Brun E., Cloutier P., Sicard-Roselli C., Fromm M., Sanche L. (2009) Damage induced to dna by low-energy $(0-30 \mathrm{eV})$ electrons under vacuum and atmospheric conditions, J. Phys. Chem. B 113 (29), 10008-10013.

Butterworth K.T. et al. (2008) Gold nanoparticles: from nanomedicine to nano sensing, Nanotechnol. Sci. Appl. 1, 4566.

Carmeliet P., Jain R.K. (2000) Angiogenesis in cancer and other diseases, Nature 407 (6801), 249-257.

Casta R., Champeaux J.P., Sence M., Moretto-Capelle P., Cafarelli P., Amsellem A., Sicard-Roselli C. (2014) Electronic emission of radio-sensitizing gold nanoparticles under X-ray irradiation: experiment and simulations, J. Nanopart. Res. 16 (4), 2348.

Chauvie S., Francis Z., Guatelli S., Incerti S., Mascialino B., Moretto P., Nieminen P., Pia M.G. (2007) Geant4 physics processes for microdosimetry simulation: design foundation and implementation of the first set of models, IEEE Transactions on Nuclear Science 54 (6), 2619-2628.

Cho S.H. (2005) Estimation of tumour dose enhancement due to gold nanoparticles during typical radiation treatments: a preliminary Monte Carlo study, Phys. Med. Biol. 50, N163-N173.

Chow J.C.L., Leung M.K.K., Jaffray D.A. (2012) Monte Carlo simulation on a gold nanoparticle irradiated by electron beams, Phys. Med. Biol. 57, 3323-3331.

Connor E.E., Mwamuka J., Gole A., Murphy C.J., Wyatt M.D. (2005) Gold nanoparticles are taken up by human cells but do not cause acute cytotoxicity, Small 1, 325-327.

Delaram P., Mahdi G., Mehdi M. (2013) Tumor dose enhancement by gold nanoparticles in a $6 \mathrm{MV}$ photon beam: a Monte Carlo study on the size effect of nanoparticles, NUKLEONIKA 58 (2), 275-280.

Garnica-Garza H. (2013) Microdosimetry of X-ray irradiated gold nanoparticles, Radiat. Prot. Dosim. 155, 59-63.

Herold D.M. et al. (2000) Gold micro-spheres: a selective technique for producing biologically effective dose enhancement, Int. J. Radiat. Biol. 76, 1357-1364.

Incerti S., Suerfu B., Xu J., Ivantchenko V., Mantero A., Brown J.M.C., Bernal M.A., Francis Z., Karamitros M., Tran H.N. (2016) Simulation of Auger electron emission from nanometersize gold targets using the Geant4 Monte Carlo simulation toolkit, Nucl. Instrum. Methods Phys. Res. B 372, 91-101.

Jones B.L., Krishnan S., Cho S.H. (2010) Estimation of microscopic dose enhancement factor around gold nanoparticles by Monte Carlo calculations, Med. Phys. 37, 3809-3816.

Kadri O., Ivanchenko V., Gharbi F., Trabelsi A. (2009) Incorporation of the Goudsmit-Saunderson electron transport theory in the Geant4 Monte Carlo code, Nucl. Instrum. Methods Phys. Res. B 267 (23-24), 3624-3632.

Lechtman E., Chattopadhyay N., Cai Z., Mashouf S., Reilly R., Pignol J.P. (2011) Implications on clinical scenario of gold nanoparticle radiosensitization in regards to photon energy, nanoparticle size, concentration and location, Phys. Med. Biol. 56 (15), 4631-4647.

Lechtman E., Mashouf S., Chattopadhyay N., Keller B., Lai P., Cai Z., Reilly R/, J-P P (2013) A Monte Carlo-based model of gold nanoparticle radiosensitization accounting for increased radiobiological effectiveness, Phys. Med. Biol. 58, 3075-3087. 
Leung M.K. et al. (2011) Irradiation of gold nanoparticles by X-rays: Monte Carlo simulation of dose enhancements and the spatial properties of the secondary electrons production, Med. Phys. 38, 624-631.

McMahon S.J., Hyland W.B., Muir M.F., Coulter J.A., Jain S., Butterworth K.T., Schettino G., Dickson G.R., Hounsell A.R., OSullivan J.M., Prise K.M., Hirst D.G., Currell F.J. (2011) Biological consequences of nanoscale energy deposition near irradiated heavy atom nanoparticles, Sci. Rep. 1, 18.

Noblet C., Chiavassa S., Smekens F., Sarrut D., Passal V., Suhard J., Lisbona A., Paris F., Delpon G. (2016) Validation of fast Monte Carlo dose calculation in small animal radiotherapy with EBT3 radiochromic films, Phys. Med. Biol. 61, 3521-3535.
Rahman W.N., Bishara N., Ackerly T., Fa He C., Jackson P., Wong C. (2009) Enhancement of radiation effects by gold nanoparticles for superficial radiation therapy, Nanomedicine: Nanotechnology, Biology, and Medicine 5, 136-142.

Ricketts K., Castoldi A., Guazzoni C., Ozkan C., Christodoulou C., Gibson A.P., Royle G.J. (2012) A quantitative X-ray detection system for gold nanoparticle tumour biomarkers, Phys. Med. Biol. 57, 5543-5555.

Sanche L. (2009) Role of secondary low energy electrons in radiobiology and chemoradiation therapy of cancer, Chem. Phys. Lett. 474, 16.

Cite this article as: F. Benlakhdar, A.S.A. Dib, A.H. Belbachir. Effect of nanomaterials on the absorbed dose during an X-ray exposure. Radioprotection 51(4), 279-285 (2016). 